$\mathbb{T}$ periodica polytechnica

\author{
Social and Management Sciences \\ $16 / 2$ (2008) 81,88 \\ doi: $10.3311 /$ pp.so.2008-2.04 \\ web: http://www.pp.bme.hu/so \\ (c) Periodica Polytechnica 2008
}

RESEARCH ARTICLE

\section{Diversification of strategic uncertainties in the business of environmental policy}

Miklós Antal

Received 2009-07-09

\begin{abstract}
Strategic uncertainties are frequently encountered in policy situations. The behavior of actors in policy networks is often unpredictable, while the success of a given actor may depend on decisions of other actors. It seems rational to presume that strategic risks are taken into consideration besides expected outcomes in policy decision making.

In the present paper we outline an analogy between stock markets and policy situations. It is conjectured that similarly to stockholders who diversify their portfolios to maximize expected returns at a given level of riskiness, policy actors also evaluate the diversity of their 'portfolio of relations' and tune the assumed risks in individual relationships accordingly. The main hypothesis of the paper is that if the number of relationships grows (viz. unpredictable variations in partners' behavior increasingly compensate each other), policy actors will assume greater risks in individual relations. Thus, we surmise that strategic risks can be diversified by relationship portfolios.

A survey of Hungarian environmental NGOs was carried out to prove the hypothesis. Statistical results confirmed our assumption at the $99 \%$ confidence level. To our knowledge, this is the first direct evidence that the boldness of a policy strategy depends on the number of partners. More generally, the novelty of the present work lies in the useful analogy between stock markets and an environmental policy situation, and the demonstrated connection between the number of partners and the assumed strategic risks.
\end{abstract}

\section{Keywords}

strategic risk $\cdot$ risk tolerance $\cdot$ stock market analogy $\cdot$ boldness of strategies $\cdot$ policy network $\cdot$ relationship portfolio

\section{Miklós Antal}

Department of Environmental Economics, BME, H-1111 Budapest, Sztoczek u. 2, Hungary

e-mail: antalmi@gmail.com

\section{Introduction}

Environmental policy problems are usually of high complexity [6]. To begin with, large amounts of scientific knowledge are needed to understand the nature of a feature environmental problem. The required data are not always easily accessible and proper modeling of phenomena can also be complicated. Second, a number of different actors with different information, knowledge, objectives, strategies and influence can be involved, interlinked by different relationships [11]. Governmental and non-governmental, for-profit and not-for-profit organizations compete or collaborate to reach their respective goals. Third, institutional settings affect the chances of different actors and outcomes of the policy process [3].

All these factors of complexity bear different sources of uncertainties. The lack of scientific knowledge is called cognitive uncertainty. Strategic uncertainty refers to the role of unpredictable behavior of a certain actor directly or indirectly affecting other actors' success. Institutional uncertainty arises, because decisions are made in different places under different circumstances at different levels of the decision making system [4].

While the role of cognitive uncertainties in decision making is an integral part of the scientific literature, the focus of the present article is on strategic uncertainties. The presented idea about the reduction of strategic hazards may also be generalized and applied to institutional uncertainties.

Strategic considerations of a given actor involved in the policy process imply risks for those partners whose success depends on the given decision. Their optimal strategy may depend on the decision of the partner - outcomes are often determined by sets of strategies. If the strategy of a partner is unknown, then it can be represented by a stochastic variable. Actors may estimate probabilities of different behaviors based on their experiences. However, the measurement of such probabilities is virtually impossible in real policy situations. The actions of given actors can not be repeated many times under the same conditions to build statistics. Moreover, complex policy situations can not be interpreted without the context in which the processes are embedded, so it is not easy to draw conclusions from comparative studies. The bottom-up approach to explain actors' behavior fails due to 
similar reasons: game theoretic models otherwise amenable to measure and predict strategic behavior in risky situations [8] are way too abstract to be applicable to real life phenomena.

Consequently, albeit the momentous role of strategic uncertainty is often highlighted in policy literature (see e.g. [1] ), its relation with a number of different factors describing the policy process (e.g. structural characteristics of the actor network) is unclear. The aim of the present paper is to shed light on the relation between strategic uncertainties and a basic structural attribute; the number of partners. If we are able to reveal such a connection, this parameter can be included in strategic planning.

Throughout the article we investigate a suggestive case where an NGO tries to change the practice of companies to improve their environmental performance. There are many ways to do so: the strategy chosen by the NGO can be friendly and cooperative, or it can be very ambitious and vehement, sometimes even aggressive. In the case of a friendlier strategy targeting minor changes, step by step progress can almost surely be expected. More aggressive strategies offer the chance of rapid changes but also raise the possibility of complete denial of any improvements. Accordingly, strategic uncertainty grows as the strategy becomes increasingly aggressive. Environmental organizations have to balance their high-flying objectives with leniency to reduce the risk of denial.

This optimization resembles the behavior of risk-averse (or loss-averse) investors in stock markets. They also search for maximum returns and try to minimize uncertainties called volatility in their case. Similarities between stock markets and strategic decision making encouraged the elaboration of the outlined analogy between the two seemingly distant fields. Section 2 is devoted to the analogy itself: notions from the two fields are matched to each other and portfolio building observed in stock markets is translated into a respective non-trivial, hitherto unknown strategic behavior. Section 3 summarizes the way we measured the effect with questions on hypothetic situations - a method borrowed from investment theory as well. Section 4 is for the statistical evaluation of the results obtained in our survey, supplemented by comments on the validity and applicability of the results. Conclusions and perspectives are given in Section 5.

\section{The analogy and the ensuing hypothesis}

\section{The analogy}

We compare stock markets and policy systems. In the stock market the subject of the investigation is a stockholder. In the policy system, we analyze an NGO that is in connection with some companies of a given branch of business. (Obviously, it is advisory to choose the simplest system that shows the studied behavior. In this case there are no complex effects of unknown origins that are typical in the case of complicated structures.) The aim of the stockholder is to maximize returns. The aim of the NGO is to maximize the environmental performance of the companies.

The means that allow the respective actors to reach their goals are buying stocks and establishing connections with companies, respectively. Thus, having stocks in the one field is matched to having connections with companies in the other.

Here we note that there is a difference between the two cases. Stockholders can change two parameters - the type of the stock that determines its riskiness and the amount they buy; while NGOs can only change the riskiness of their strategy, they either have one connection or not, there is no intermediate case. However, if we imagine a hypothetical stock market, where investors can either purchase a stock for a given sum or they can not buy any of it, their incentives to build portfolios - the central topic of the present paper expanded later - are not eliminated. So, in such a "discretized" market, where stock amounts can not be freely changed, investors still build portfolios to reduce risks at a certain level of expected returns [12]. They seek for the least imperfect combinations where expected returns are high and volatility is low. Fig. 1] shows a very simple example of diversification in a "discretized market" with a discretized efficient frontier (only the black dots are possible states). E.g. holding Stock 2 is suboptimal, so there are investors with given risk-aversion who diversify their portfolios. (The goal of diversification is to jump up and left from point to point in the expected return - volatility graph.)

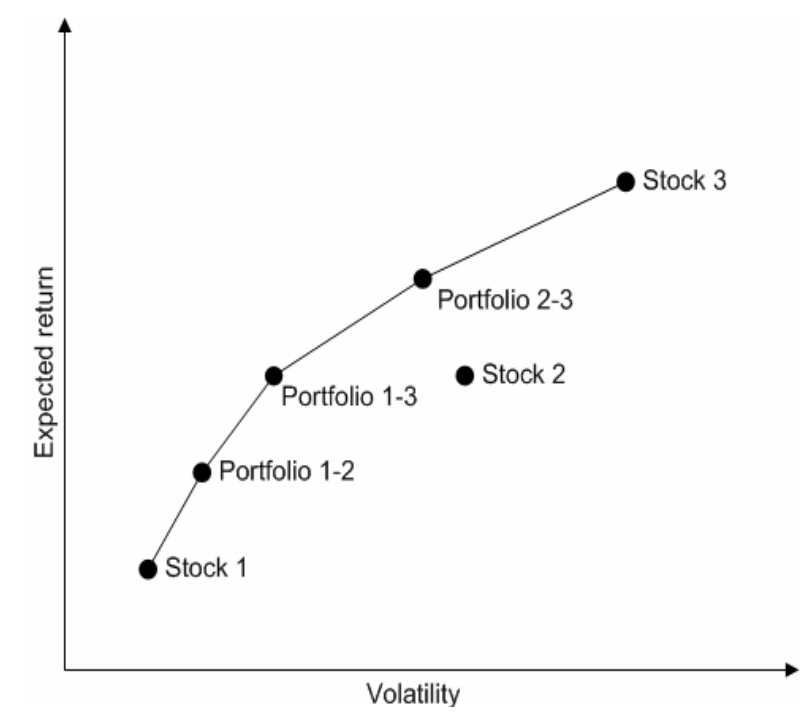

Fig. 1. Diversification in a "discretized market"

Success is determined by the returns of the stock and the behavior of the company, respectively. The analogy is sound, because the factor that measures success is a stochastic variable determined by the complex behavior of the whole system of the stock market or the policy arena. (Moreover, if an investor has several different stocks or an NGO has several different relations, returns are positively correlated. These changes in similar directions are caused by, inter alia, macro-economic effects and peer pressure, respectively.)

The assumed risks are given by the bought stock itself in stock markets and the boldness of the strategy in NGO-company relations. The analogy is clear: the bigger the assumed risk is, the higher the expected returns are. In concrete: more volatile 
stocks have higher expected returns [15] and bolder (and thus riskier) strategies are used to achieve greater results. (Arguably, the boldness of the strategy is correlated with strategic risks: the bolder the strategy is, the bigger is the chance that the proposal will be denied. However, in case of success, bolder strategies deliver better results than less daring ones.) For a summary of the analogies see Table 1 .

Although, the fact that there is no unequivocal value of the assumed risk in the policy case leaves two opportunities open. Either the curve of expected returns against boldness has a maximum and NGOs try to find this point, viz. they maximize expected returns; or expected returns and strategic risks are evaluated together by risk-averse (or loss-averse) NGOs. Notwithstanding that the expected returns fall if NGOs become too aggressive (i.e. expected returns, indeed, have a maximum as a function of boldness); it is still possible that strategic uncertainties are also considered viz. indifference curves are not horizontal in Fig.2.

Thus, we need to distinguish risk-averse behavior from the sheer maximization of expected returns. To tell apart riskneutral and risk-averse behavior we look at portfolios. Similarly to stock markets, where portfolios are built to diversify risks, it is possible that NGOs evaluate expected results and strategic uncertainties together. In stock markets, investors build diverse portfolios and strive to reduce volatility at a given level of expected returns by holding different stocks so that oppositely directed fluctuations in returns compensate each other. Alternatively, this is the way how at a given level of volatility expected returns can be maximized.

If the case is similar in the policy arena, the boldness of NGO's chosen strategy can be affected by the constitution of their 'portfolio of relations'. If the number of the companies they try to affect grows, variations in their behavior (defining the given NGO's success) will compensate each other - exactly as the volatility of portfolios decreases as the number of stocks in the portfolio (whose returns are not fully correlated) grows. Hence, a similar reduction in strategic uncertainties is possible. However, if NGOs were risk-neutral, they would not care about strategic uncertainties and they would use the same strategy regardless of the number of the partners to maximize expected returns in individual relations.

The main hypothesis of the present paper is the following: if the number of the companies, with which a given NGO is connected, grows, its strategy to influence their behavior will become bolder. More generally, we conjecture that actors in the business of policy are risk-averse and they can be characterized by a certain level of risk tolerance. Consequently, if strategic uncertainty falls (as the number of partners grows) their response will be to assume greater risks in their individual relations. The adjustment of the assumed risks, the treatment of uncertainties is similar to stockholders' behavior in stock markets: policy actors diversify strategic risks.

\section{Preliminary remarks}

Before going into details about the proof of the hypothesis, we list some notable remarks. First, unlike investors who can freely choose their portfolio elements, the required connections in policy situations are sometimes given. Resources like powers, status, legitimacy, knowledge, information, or money may necessitate the inclusion of given actors [11]. In the exemplary case of the environmental performance of businesses, NGOs may try to influence all companies in a given area, so their opportunities to choose partners can be strongly restricted. However, that means no restrictions in terms of the assumed risk, because riskiness can be tuned by the chosen strategies. So, the outlined analogy is not distorted.

Second, if we are talking about an NGO and its several relationships, the boldness of strategies can be different in different relations. From a theoretical point of view, the clearest form of our hypothesis can be formulated for those partners whose approach is unknown, because then there is no reason to pursue different strategies in different relations. Though, strategies of the NGO can be significantly different if partners are known to be cooperative, indifferent or hostile. Nevertheless, it is always possible (independently of the approach of the partners) to change the assumed strategic risk according to the number of partners. The only difference is that it is more difficult to discern the effect of changes in strategic uncertainties if there are other strategic concerns.

More generally, a third concern is that there are a number of other factors that influence the boldness of the strategy. Obviously, the public acceptance of the goals of the NGO, the complexity of the issue strongly linked to its marketability, the available time, power relations and several other factors affect strategic decisions. However, the fact that the number of partners is not always decisive in strategic considerations does not mean that its role is negligible. Our aim was to separate the effects of the number of connections and demonstrate that it is indeed a constituent in strategic decision making.

Risk theory - basic dilemmas and methods of measurement

Since we wish to perform measurements regarding behavior under uncertainties, exact measurement instructions are needed. These instructions are based on a theory - we need to define the parameter under study. Conclusions drawn from empirical results are theory based, too. Consequently, we outline basic questions of risk theory - originally developed to describe behavior of actors in stock markets - so that we can ask proper questions and evaluate results in policy situations.

It is generally assumed in modern portfolio theory that investors try to avoid risk. The original theory interpreted risk aversion by assessing the expected utility of wealth before and after an investment. The concave utility function of wealth was deemed to be responsible for risk aversion [2].

Recently it was unequivocally shown that Markowitz's origi- 
Tab. 1. Analogies between stock markets and policy situations

\begin{tabular}{lll}
\hline & Stock market & Policy situation \\
\hline Subject of the study & a stockholder & an NGO \\
Risky means of actions & stocks & relationships \\
Success factor & stock returns & company behavior \\
Assumed risk (single actions) & stock volatility & boldness of the strategy \\
A factor in portfolio building & number of different stocks & number of relationships? \\
Ensuing strategy & diversification & ??? \\
\hline
\end{tabular}

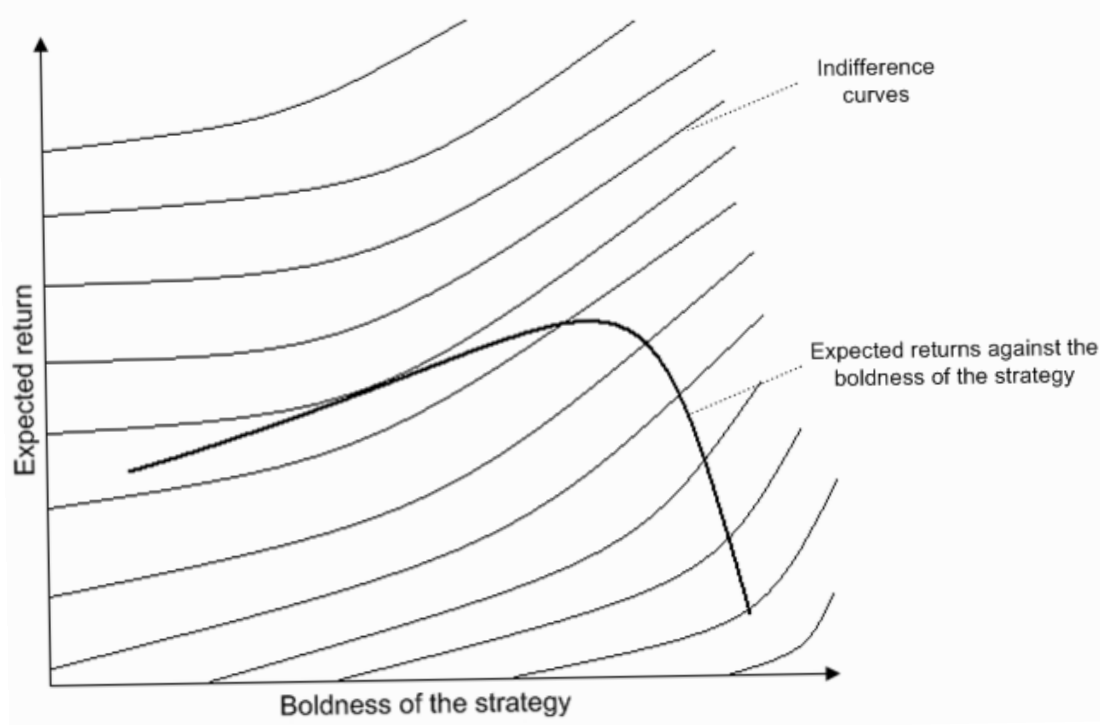

Fig. 2. Expected returns against the boldness of the strategy (thick line), and the indifference curves of a given policy decision maker

nal theory and its later versions give infinitely high risk aversion for high values and total risk neutrality at lower values of wealth [14]. This contradicts real world observations: we buy lottery tickets and cheap insurances. Accordingly, the emphasis was shifted to the theory that regards the expected utility of income (viz. potential changes in wealth) as the fundamental variable. Instead of the initial and final values, the potential positive and negative changes in wealth were compared to calculate the expected utility of income and make a decision in a risky situation [5]. At the same time, loss aversion (originally described by Kahnemann and Tversky, 1979 [10]) was proposed instead of risk aversion as the organizing principle of actions in risky situations [13].

However, problems of the new model and contradictions with portfolio theory (e.g. portfolio-independent investments) were realized soon (e.g. [9]). Hybrid models were elaborated to incorporate all observed features into a single model. However, up to now, there is no convenient, universally accepted model to describe risk or loss aversion.

On the other hand, what is common in all these models is that actors react to uncertainties with changes in their behavior. These changes are actor dependent and researchers try to represent actor-specific attributes with risk tolerance or loss tolerance factors, whose measurement methods are quite similar. Fortunately, the effect we would like to demonstrate is analo- gous to this common point of the theories: right now, our aim is to show how behavior changes in policy situations under uncertain conditions. The clarification of causes, answers to the why questions are tasks of the future. However, similarities between financial and political decisions could one day allow potential breakthroughs in the one field to have seminal consequences in the other. Nevertheless, as for now, we should turn our attention to the measurement techniques applied to investigate behavior under risk.

As articulated earlier, we surmise that actors in the world of policy can also be characterized by a certain level of risk tolerance. Hanna et al. observed that there are at least four methods of measuring risk tolerance [7] : asking about investment choices, asking a combination of investment and subjective questions, assessing actual behavior, and asking questions based on hypothetical scenarios.

As stated in the introductory part, assessment of actual behavior is not a very promising opportunity in the case of policy. Asking about real life choices can also result in distorted results, because we can not study a sufficiently large sample to level out other effects stemming from different factors that influence behavior. Thus, we chose hypothetical scenarios as a measurement method. Here the role of the number of the actors can be investigated in a 'pure' form and the sample of the query is not restricted to organizations that have already encountered cases in 
which they changed their strategies due to changes in the number of their partners. Details of our survey and its results are given in the next section.

\section{The survey}

\section{Surveyed organizations}

Our aim was to study the behavior of NGOs that pursue environmental activities and see how their strategies change if there are changes in the composition of their 'portfolio of relations'. We looked for NGOs that may get in touch with companies and try to affect their behavior. We restricted the scope of investigations to Hungarian NGOs for two reasons. First, the fact that we studied organizations in a single country allowed us to further reduce the number of parameters (social settings, organizational culture etc.) that could have affected strategic considerations. Second, it was easier to reach Hungarian organizations and we could endeavor to contact nearly all of the environmental NGOs in the country.

We used the comprehensive web page "nonprofit.hu" to find NGOs. We searched for NGOs that marked environmental protection as one of their activities. We listed 253 different organizations that had proper electronic contact information, actualized their data in the last two years, and could receive $1 \%$ tax donations (implying that they were more or less serious organizations). As there were some umbrella organizations among these, the original list represented approximately 300 organizations. We sent out a survey electronically (as an attached document) with 9 questions to all of these NGOs. Unfortunately, 47 email addresses turned out to be incorrect or unavailable. Eventually, even after we repeated the query, we received meager 30 completed surveys. Thus, we targeted the inactive NGOs with an email consisting of only the most crucial question and asked them to reply in one word. These renewed efforts paid off in the form of 28 more answers.

Hence, we could build statistics from 58 separate answers from 58 different NGOs. However, we had only one question to rely on; supplementary questions included in the original survey could only be used as weaker guidance based on the first 30 results.

\section{Survey questions}

In the original survey we asked 9 questions (see the whole survey in the Appendix). Apart from our central questions (1-3) we asked questions about the validity of our theory (4-5) and tried to gain information about other factors (approach and status of the partner) influencing strategic considerations (6-8). Finally, we asked about real-life examples.

After assessing the first 30 results, we saw that our hypothesis is likely to be confirmed. Out of the first three questions, we chose the one that seemed to be most common in reality: the case when NGOs try to influence companies (Question 1 in our survey):
Suppose that your NGO would like to affect some companies in a given branch of business so that they improve their environmental performance. Depending on the number of companies, how would you change your strategy? Would you shift to bolder or to more cautious strategy if you tried to influence more companies?

\section{We would not change our strategy.}

\section{We would use bolder strategy.}

\section{We would use more cautious strategy.}

We emailed this question to our list again and received 28 further answers. As it is obvious from the question, our goal was to reveal the mechanism of risk aversion in the policy case and we did not strive to render quantitative parameters to the observed behavior. Arguably, statistical evaluation of the answers can shed light on the altered behavior of policy actors under risk.

\section{Results}

In the case the fundamental question 32 of 58 respondents chose bolder strategies, 17 organizations would not change their strategy, and only 9 organizations answered that they would apply more cautious strategies.

Supplemented by the data collected from full surveys, results are given in Table 2 (Question 2 and 3 were similar to the one above, the only difference was that instead of companies partners were decision makers and other organizations, respectively.)

Tab. 2. Results: strategic changes caused by changes in the number of partners

\begin{tabular}{llll}
\hline & "no changes" & "bolder" & "more cautious" \\
\hline Complete results & $17(29,3 \%)$ & $32(55,2 \%)$ & $9(15,5 \%)$ \\
Survey Question 1 & $12(40,0 \%)$ & $15(50,0 \%)$ & $3(10,0 \%)$ \\
Survey Question 2 & $8(26,7 \%)$ & $14(46,7 \%)$ & $8(26,7 \%)$ \\
Survey Question 3 & $10(33,3 \%)$ & $14(44,7 \%)$ & $6(20,0 \%)$ \\
\hline
\end{tabular}

\section{Evaluation}

\section{The central hypothesis}

To begin the evaluation, we focus on the central question posed in the previous section. As there were three choices, we can use a modified version of binomial analysis. In the case of a random distribution of the answers, the probability of each answer is $1 / 3$. The probability that there are $x$ answers from one type out of the 58 answers is:

$$
P(x)=\left(\begin{array}{c}
58 \\
x
\end{array}\right) \cdot\left(\frac{1}{3}\right)^{x} \cdot\left(\frac{2}{3}\right)^{58-x}=\frac{58 !}{x ! \cdot(58-x) !} \cdot \frac{2^{58-x}}{3^{58}} .
$$

Our aim is to see that the number of positive answers (bolder strategies) is too high and the number of negative answers (more cautious strategies) is too low to be coincidental. We could arrive at such statements if we calculated the confidence intervals 
at the $99 \%$ confidence level. So, the task is to calculate $N$ and $M$ so that $\sum_{x=1}^{N} P(x) \geq 0,99$ and $\sum_{x=M}^{58} P(x) \geq 0,99$. Numerical calculations give $N=28$ and $M=11$.

As our results (32 and 9) are not in the $99 \%$ confidence intervals (1..28 and $11 \ldots 58$, respectively), we have to reject the assumption that we sampled a random distribution at the $99 \%$ confidence level.

Consequently, our hypothesis that policy actors typically shift to bolder strategies if the number of their partners grows, can be accepted at the $99 \%$ confidence level. Both the particularly high proportions of positive answers and the particularly low proportions of negative answers corroborate the central hypothesis of this paper.

(The statistical analysis was repeated with the statistical software SPSS 15. Results obtained with its Binomial Test were perfectly the same that we got manually from the above calculations.)

Similar but weaker statements hold for the 30 original survey results. The proportion of positive answers lies out of the $90 \%$ confidence interval for all of the first 3 questions. Except for question no. 2 , the low proportion of negative results can also be accepted to be non-accidental at the $90 \%$ confidence level.

\section{Validity and applicability}

In order to get rid of ambiguities, we have to point out some implicit assumptions and make a few more remarks. First, throughout the query we implicitly assumed that unidirectional changes of the number of partners shift strategies in the same direction, viz. there are no distinguished numbers of partners where these trends turn to their opposites. However evident this claim may look, we asked a question about it in our survey. 21 of 30 respondents confirmed that this implicit assumption was right, while most of those who expressed negative opinions did not fully understand the question as it became obvious from their concerns. Until now, we have not found a reason why strategic shifts would abruptly turn to their opposites if the changes in the number of partners continued in the same direction, so we do not deem this assumption to be problematic.

Second, we may conjecture that an NGO applies the same strategy in its relations with different partners whose status and role in the given case is identical. If partners are unknown, this may be a rational choice. Furthermore, sometimes it is important to apply the same strategy in different relations to be and look fair. However, as 12 of 30 respondents pointed out, it is possible that other factors (e.g. the known approach of given actors) influence strategies and the conjecture does not hold. Here we have to emphasize again that it does not cause problems with the theory: different strategies may be shifted simultaneously due to changes in the number of actors, no matter how variant they are. The only effect is that it will be more difficult to reveal the phenomenon under study if there are other major strategic concerns.
Third, the sample size was not sufficiently large to draw conclusions regarding the role of the following factors: the NGO's size, the role or the status of the partners (companies, decision makers, organizations; important or less important roles in the case), or the approach of the partners (positive, negative, neutral, unknown). Further studies are needed to clarify the effects attributed to these factors.

Fourth, our analysis studied NGOs with environmental objectives, but we are convinced that the demonstrated phenomenon is much more general. Probably it is not always so easy to construct proper hypothetical cases as it was in the outlined investigation, but it seems to be plausible that the underlying mechanisms are the same in a wide range of policy situations. Consequently, the demonstrated research is deemed to be a manifestation of a general risk (or loss) avoiding behavior characterizing actors in the world of policy. The presented theory has thus practical implications for a variety of actors in the political battlefield.

Fifth, it is also possible that policy actors do not only diversify strategic risks, but also they use the same method to reduce institutional uncertainties. That could be a reason for political actors to have strong ties to other actors being active in different policy arenas (e.g. it can be profitable for moderate politicians to have connections to radical NGOs). However, the thorough analysis of institutional uncertainties from a diversification point of view is only a future opportunity to date.

\section{Conclusions and perspectives}

In this article we have formulated an analogy between stock markets and policy situations. The foundational idea of the paper was that actors in such different contexts react to uncertainties in similar fashions. Expected returns and risks are evaluated together to optimize behavior. If there are simultaneous efforts to achieve success (an investor holds more different stocks or a policy actor has more different relations), then fluctuations in outcomes can compensate each other. Thus, diversification offers the chance to minimize uncertainties at a given level of expected returns, or to maximize returns at a given level of uncertainties. Actors in both fields tend to make use of such opportunities. However, while the phenomenon has been well known in stock markets for more than five decades now, diversification of strategic uncertainties has not been identified before.

The revealed analogy is tested on the sample of Hungarian environmental NGOs. The hypothesis that the composition of the actor network is related to the assumed risk in individual relations is analyzed by hypothetical questions. The central argument that supports the hypothesis of diversification - the assumption that a growing number of partners inspires NGOs to apply bolder strategies to improve their environmental performance - can be accepted at the $99 \%$ confidence level.

The observed behavior in risky situations may help to include a new aspect - the number of partners - into strategic considerations. This hitherto unconsciously applied factor in deci- 
sion making can be incorporated into strategic planning. On the longer run, further steps can be made to clarify the role of policy network structures in strategic considerations. In a similar way to economics, attempts can be made to render parameters to risk or loss avoidance and quantify different actors' approach to risky situations. However, problems encountered in economics are likely to occur in the policy case as well, and additional difficulties are also expected due to worse measurability opportunities.

Nevertheless, we hope that both the theoretical construct which serves as the base of the analogy and the conclusions drawn from the survey of environmental NGOs can be beneficial - not only in the business of environmental policy.

\section{References}

1 Allaire Y, Firsirotu M, Coping with Strategic Uncertainty, Sloan Management Review 30/3 (1989), 7-16.

2 Arrow K J, Essays in the Theory of Risk Bearing, Chicago IL: Markham Publishing Company, 1971.

3 Briassoulis $\mathbf{H}$, The institutional complexity of environmental policy and planning problems: the example of Mediterranean desertification, Journal of Environmental Planning and Management 47/1 (2004), 115-35, DOI 10.1080/0964056042000189835.

4 van Bueren E M, Klijn EH, Koppenjan J F M, Dealing with wicked problems in Networks Analysing an Environmental Debate from a Networks Perspective, Journal of Public Administration Research and Theory 13/2, 193 212, DOI 10.1093/jopart/mug017.

5 Cox J C, Sadiraj V, Small-and large-stakes risk aversion: Implications of concavity calibration for decision theory, Games and Economic Behavior 56/1 (2006), 45-60, DOI 10.1016/j.geb.2005.08.001.

6 Funtowicz S O, Martinez-Alier J, Munda G, Ravetz J R, Information tools for environmental policy under conditions of complexity, Environmental Issues Series 9 (1999). European Environment Agency.

7 Hanna S D, Gutter M S, Fan J X, A measure of risk tolerance based on economic theory, Financial Counseling and Planning 12/2 (2001), 53-60.

8 Heinemann F, Nagel R, Ockenfels P, Measuring Strategic Uncertainties in Coordination Games, Vol. 804, Department of Economics and Business, University Pompeu Fabra, 2004.

9 Heinemann F, Measuring Risk Aversion and the Wealth Effect, Vol. 156, Governance and Efficiency of Economic Systems, TU Berlin, 2005.

10 Kahneman D, Tversky A, Prospect Theory: An Analysis of Decision Under Risk, Econometrica 47/2 (1979), 263-91.

11 Klijn EH, Koppenjan J, Termeer K, Managing Networks in the Public Sector: A Theoretical Study of Management Strategies in Policy Networks, Journal of Public Administration 73/3 (1995), 437-454.

12 Markowitz HM, Portfolio Selection, Journal of Finance 7/1 (1952), 77-91.

13 Rabin M, Risk Aversion and Expected-Utility Theory: A Calibration Theorem, Econometrica 68/5 (2000), 1281-1292.

14 Rabin M, Thaler R H, Anomalies - Risk Aversion, Journal of Economic Perspectives 15/1 (2001), 219-32.

15 Sharpe W F, Capital asset prices: A theory of market equilibrium under conditions of risk, Journal of Finance 19/3 (1964), 425-442, DOI $10.2307 / 2977928$.

\section{Appendix}

\section{Behavior under risk - a study of environmental NGOs}

\section{Introduction}

In the following we ask questions about strategic decisions of NGOs pursuing environmental activities. The aim of the study is to analyze opinions of the surveyed organizations about risky situations. We examine cases in which environmentalists try to change the behavior of certain economic, social or political actors (e.g. companies, schools, local governments, decision makers etc.).

The more or less unpredictable behavior of the actors they try to affect means risk for the NGOs. Depending on how ambitious their goals are and how vehement they are in persuasion, they can expect different results. While small step strategies offer minor changes with very good chances; bolder plans and stronger actions may result in significant changes for the better, but at the same time chances of a complete denial of suggestions grow. In the following we would like to study how the boldness/cautiousness of the strategy is related to the number of partners.

The survey is sent to a broad range of Hungarian NGOs pursuing environmental activities. Results obtained in the study may help to make strategic decisions and could contribute to the vitalization of our civil society. Outcomes of the statistical evaluation will be sent to all respondents without specifying concrete organizations.

If you have any questions or comments, please indicate them in the Comments section.

Thank you for your kind assistance!

\section{Survey}

Throughout the survey, we use the term cautious strategy, if the NGO is in partnership with the organization or person it tries to affect. Continuous efforts are made to reach agreement, the good relationship is very important for the NGO. Steps of actions are usually planned jointly, small step targets are set, and slow, gradual improvement is envisaged.

We use the term bold strategy, if the NGO shifts to stronger initiatives, exerts pressure, or tries to coerce its partners to improve their environmental performance. High-flying aims of bolder strategies may deliver step-like changes and significant improvements, but chances of conflicts also grow that can hamper development.

\section{Basic data}

Name of the NGO:

Number of employees:

Approximate number of volunteers:

\section{Questions}

Please underline the answer that is characteristic for your organization!

Question 1

Suppose that your NGO would like to affect some companies in a given branch of business so that they improve their environ- 
mental performance. Depending on the number of companies, how would you change your strategy? Would you shift to bolder or to more cautious strategy if you tried to influence more companies?

We would not change our strategy.

We would use bolder strategy.

We would use more cautious strategy.

Question 2

Suppose that your NGO would like to shape the opinion of some decision makers in a given decision making panel. Depending on the number of the decision makers, how would you change your strategy? Would you shift to bolder or to more cautious strategy if you tried to influence more decision makers?

We would not change our strategy.

We would use bolder strategy.

We would use more cautious strategy.

\section{Question 3}

Suppose that your NGO would like to affect the behavior of some organizations. Depending on the number of organizations, how would you change your strategy? Would you shift to bolder or to more cautious strategy if you tried to influence more organizations?

We would not change our strategy.

We would use bolder strategy.

We would use more cautious strategy.

\section{Question 4}

Until now we conjectured that (leaving all other parameters unchanged) unidirectional changes in the number of partners (increase or decrease) shifts the strategy into the same direction (so that it becomes bolder or more cautious). Is this conjecture right?

Yes.

No. (Please briefly specify causes!)

\section{Question 5}

Until now we conjectured that your NGO applies the same strategy in its relations with different partners whose status and role in the given case is identical. Is this conjecture right?

Yes.

No. (Please briefly specify causes!)

\section{Question 6}

In which case would you choose bolder strategy: if the partner's approach was positive / negative / neutral / unknown? Please write the words "positive", "negative", "neutral", and "unknown" next to the numbers. Number 1 means the most cautious, number 4 the boldest strategy.

1.

2.

3.

4.

\section{Question 7}

If the approach of the partner was unknown, in which case would you choose bolder strategies: if you tried to influence companies / decision makers / organizations? If the order is unequivocal, please put the words "company", "decision maker", and "organization" next to the numbers. (Number 1 should be the most cautious, number 3 the boldest.) If other parameters determine the boldness of the strategy, please specify it briefly!

Order:

1.

2.

3.

No unequivocal order, it depends on:

Question 8

Depending on the perceived importance of an actor in a given case, how would you tune your strategies?

We would use bolder strategies in more important relations.

We would use more cautious strategies in more important relations.

We would use the same strategy in all our relations.

Question 9

Have you ever encountered a case in the life of your NGO that changes in the number of partners lead to changes in your strategies? If yes, please briefly elicit the case!

\section{Comments}

Thank you very much for your assistance! 